\title{
Performance Evaluation of Storage Area Network(SAN)With internet Small Computer System Interface (iSCSI)For Local System PC
}

\author{
Muthanna Mohammad AL-aswad ${ }^{(1)^{*}}$, PHD.KHALIL ALWAJEH ${ }^{(2)}$ \\ (1), (2) Faculty of Computer Science \& Information Systems, Dhamar University , Dhamar city , \\ Yemen \\ Corresponding author: mothanaalaswad@gmail.com
}

\begin{abstract}
SCSI is a newly emerging protocol with the goal of implementing the Storage Area Network (SAN) technology over TCP/IP, where enables to access to remote data that in attached storage disks storages Direct Attached Storage(DAS) over IP-networked. Also it's brings economy and convenience whereas it also raises performance and reliability issues.

This paper investigates about possibility, using storage technology of the SANs, and iSCSI-SAN protocol,in local system PC, to improve access to attached storage disks storages in local system, with using iSCSISANs as virtual storage, is rather than DAS storage in local system of PC. Explicates after experiment procedure is that improving throughput of iSCSI-SANs was better than attached storage disks storages - DAS in local system. This means is that it can use iSCSI-SANs in local system of PC as attache storage disks storages as DAS, without cost, high performance, and easy control.
\end{abstract}

Keywords: SAN, iSCSI-SANs, DAS, iSCSI-SAN Protocol, SCVM.

\section{INTRODUCTION}

\subsection{Introduction}

The introduction section must contain theory related to In the past few years have seen a tremendous growth in the amount of data. Huge amounts of new data are generated by humans every year. This is because of the humans and applications uses are huge amounts of data in various format including texts, video, audio and images. There are also increasing technological revolutions on the industry, finance, education, medicine, government and most business applications. All these factors increase the need for bandwidth in networks[1], and increase the need for large storage repositories, for storage of this large data. Single attached disks and old storage technologies cannot manage the storing these amounts of data[2]. DAS is using the Small Computer System Interface (SCSI) commands. However DAS with SCSI, has some limitations from these limitations. It have not, high speed, high capacity, high availability and scalable, was storage of data are done over local system or over networks. Until emerged of studies new, are said, enables from the access to remote data that in attached storage disks storagesDAS over IP-networked, with storage technology of the SANs, and with using iSCSI-SAN protocol $[2,3,4,5,6,7]$. This are paper will be studying are (SANs, iSCSI protocol) and paper investigates about possibility, the improving of access to attached storage disks storages that in local system for PC, with using using storage technology of the SANs, and internet SCSISAN ( iSCSI- SAN) protocol. Where will be using SANs as virtual storage iSCSI-SANs is rather than DAS storage in same local system for PC. The Storage Concentrator Virtual Machine (SCVM), creates iSCSISANs storage as virtual storage. It provides an advanced, fully featured iSCSI-SAN Storage within an SAN Server [3]. From through do are conversion attached storage disks storages-DAS to a virtual storages are called iSCSI-SANs. The reaching to iSCSISANs is procure with using iSCSI-SAN protocol, over IP-networked. Where focused about evaluation of performance of iSCSISANs as storage system is using in local system, and is compare it with DAS storage, in same system, with new benchmark tool called IOMeter [8]. Clear after experiment procedure is that improving throughput of iSCSI-SANs in terms of (l/Os, MB/s), and average response time in different block sizes of the $(1 / O)$ request, was better than DAS in local system PC. The performance of iSCSI-SANs is better than DAS in local system for PC, and was satisfactory. 


\subsection{Motivation}

All storage are captive to an individual PC or computer platform, such as a laptop, a desktop PC, or a single server in a small business, disk storage usually resides inside the processor enclosure and is a simple form of DAS. DAS devices depend on the host OS to handle I/O. SCSI is used as a connection medium in DAS and carries and controls the blocks between the host and attached device. However DAS and SCSI has some limitations these limitations, scalability is one of the them, the other limitation in SCSI is low number of devices that can be serviced at the time, the maximum number of attached devices to bus topology can be support by SCSI is around fifteen, because of affecting to the performance of the system these number can be decrease to four or five. The other limitation on DAS, was the availability and reliability of the system. In DAS, all DAS devices share the same cable. With the numbers of DAS devices constantly increasing, the cable between DAS and host may be very busy. Thus, the system performance is affected. In SCSI, because of the large number of cables the probability of system failure is also high and any failure in cables that connects to the storage devices can cause a system failure and lose the connectivity and data to applications. The other limitations of SCSI protocol is related to the speed and distance that can be supported by this protocol, the maximum of 25 meters long distance makes the SCSI protocol not a convenient solution for long distances[2].

All this the problems of DAS are making from DAS not the suitable storage for be the storage system very much vital, has high speed, high capacity, high availability and scalable, was storage of data are done over local system such as PC or over networks. But emerging studies new as will mentioned later in related studies, to is solve and enable from access to remote data that in DAS storages over IP-networked, with using storage technology of the SANs, and iSCSISAN protocol [2,3,4,5,6,7], as shown in "fig. $2 "$.

The paper aims at improving the storage in local system of PC, with the help of iSCSI protocols and SANs technology, DAS can be efficiently utilized, in local system of PC, to try the improve of performance of storage in it, where will using the iSCSI-SANs and iSCSI protocol is rather than DAS storage that is using SCSI protocol, and as well, this paper studies SANs, iSCSI protocol.

\subsection{Limitations that were limiting from SAN}

Presently, SAN are widely used, and have become the most important solution to improve storage performance and reliability. The storage networks in SAN systems commonly use fiber networks and fiber channel (FC) disks as storage medium[9].

However, the high cost of fiber networks and fiber disks make SAN expensive. In addition, the difficulties in controlling and expanding fiber disk arrays make it hard for customers to include their present disk arrays into the SAN systems, thereby wasting existing resources. These problems limit the application of SAN systems [9]. So recently, the emerging of iSCSI protocol, which approved by the Internet Engineering Task Force(IETF) in 2003. iSCSI-SANs operate at 1 Gbps Ethernet speeds, though this could increase to 10 Gigabit Ethernet (GBE) as existing Network Interface Cards(NICs) and switches are upgraded to accommodate 10 GigE [10].

The " fig. 1" is showing uses of iSCSI protocol, as gateway to enables applications on an IP network to use an iSCSI Initiator to connect to FC Targets. The iSCSI gateway translates iSCSI protocol to FC Protocol (FCP), bridging the IP network and FC-SAN [12].

To providing a SAN over the $10 \mathrm{~Gb} / \mathrm{Sec}$, iSCSI makes it available to use the highspeed and high performance devices over the IP infrastructure, use the low cost IPbased devices such as switches and routers are closely compete with FC in terms of performance, speed and cost[2]. IP SAN is also known as iSCSI-SAN for the protocol it employs, the names are generally interchangeable for this discussion. The development of an iSCSI protocol for an SAN environment that uses a common SCSI protocol to replace an FC protocol to be can significantly improve the SAN's performance/price ratio and flexibility [9]. 


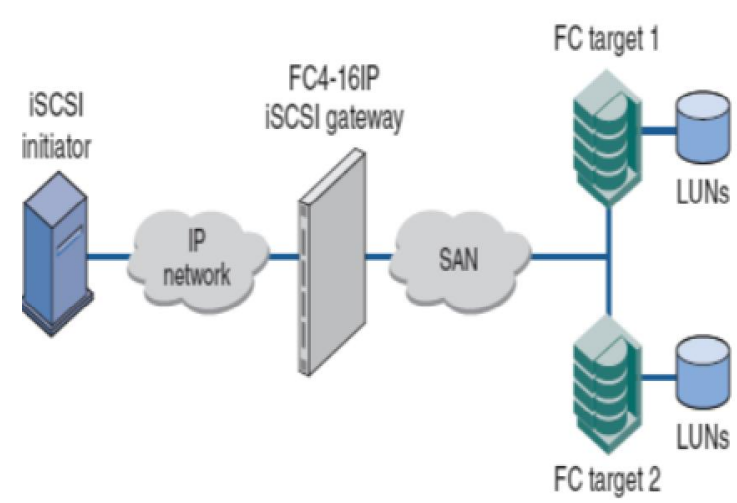

Fig. 1 Shows A basic iSCSI Gateway Servicelmplementation [12]

While studies new is emerging as will about ,[2,3,4,5,6,7]mentioned later in -possibility from building prototype iSCSI attached SANs as virtual storage with using and,storage disks storages like DAS storage and, with storage technology of the SANs for access to ,SAN protocol-with using iSCSI remote data that in attached storage disks as shown in ,networked-storages over IP .2 .Fig"

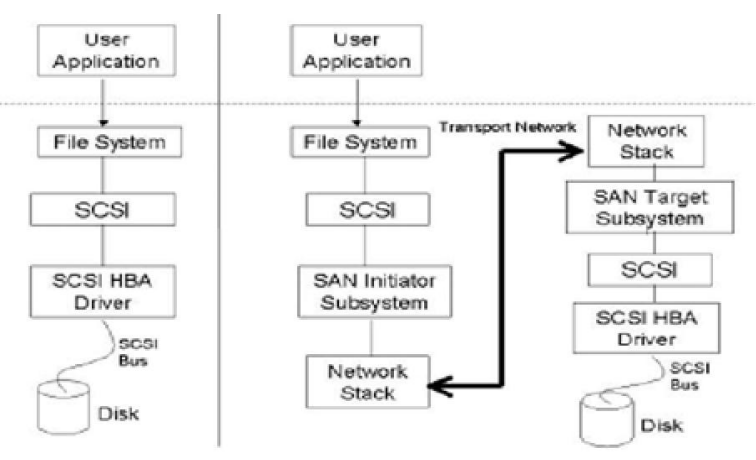

Fig. 2A mapping of the DAS over the IPNetworked Using iSCSI-SAN Protocol.

\subsection{Goal}

Goal of the paper are improving of storage in local system of PC, and which can achieves in the following points :

1. The access to DAS with storage technology of the SANs in local system.

2. Increasing of the processing speed to achieve high $\mathrm{I} / \mathrm{O}$ performance.

3. Regenerate the old idle hard disks for PCs.

4. The users can add more disks in the PCs to provide large storage space.

5. Save the money which will be used to buy a storages of SAN and connection designing.

6. Data storage backup is simplifies.
7. iSCSI-SANs provisioning and storage tiering optimizes the utilization of storage infrastructure.

8. Application installation does not cause any changes to the operating system and the file system.

9. Easily manage the iSCSI-SANs system.

10. Eliminate the need for new technical training for this service.

\subsection{Related studies}

Define abbreviations and acronyms the first Several studies have focused on improvements of the iSCSI protocol or on evaluates the performance of the iSCSI-SAN protocol and iSCSI-SANs under different scenarios:

After emerging the Storage technique over iSCSI-SANs based on the iSCSI protocol that make GBE network works in service of the FC network. In same time emerged several from studies that was about possibility that enable from improve work of iSCSI protocol from where increase of performance, reliability, synchronous, asynchronous etc. Where discussed Kalman $Z$, the design considerations and decisions in defining the iSCSI protocol, discussed some of the issues that were addressed in designing the iSCSI protocol, why use TCP, how multiple TCP connections can be used to increase performance and reliability. Discuss the reasons for the design choices on each of these issues [13].

Withal C.M. Gauger et al. proposed a models for iSCSI write requests over TCP/IP networks, e.g., as used in asynchronous mirroring applications. This model evaluated with the session under a realistic request traffic model with and without interleaving. In this evaluation, among the impact of Round Transmission Time (RTT) on the maximum throughput and the duration of the write request, the trade-off between network and processing power has been pointed out [14].

A. Pallampati et al, provided technology for Remote Direct Memory Access (RDMA) by Internet Wide Area RDMA Protocol (iWARP), which runs over TCP/IP networks provides efficient data transfers. Where was performance evaluation of iSCSI over RDMA, was done successfully, and the process that maps iSCSI protocol over the iWARP protocol suite [15].

D. Zhang et al, focused primarily on a performance study with comparing Stream 
Control Transmission Protocol(SCTP) and TCP as the underlying transport layer protocol for iSCSI. Where were the results of simulation and performance analysis indicate that iSCSI over SCTP has advantages over TCP [16].

In [17] et al ,was focused on a pair of dominant external storage technologies from the IT world, both now available in IP network format to be employed with DVRs, NVRs and IP cameras. The two solutions are iSCSI-SAN and Network Attached Storage (NAS). While the names may sound somewhat similar, but they produce very different results. In this document after cases examines why iSCSISAN is clearly, the result were that it iSCSISAN is the better choice for video surveillance [17].

He Jun Wang Li et al, worked on iSCSI security mechanisms, they improve the performance by a secure iSCSI scheme based on SSL. They implemented iSCSI system by adding SSL functions to iSCSI protocol. The results of this experiment shows that throughput of the security iSCSI based on SSL increased $25 \%$ and CPU utilization rate decreased $50 \%$, when compared to secure iSCSI based on IPSec [18].

$\mathrm{Xu}$ Xuedong et al. proposed and realize a SAN storage and encryption system, it is based on iSCSI technology to achieve a static data encryption, i.e., proposed the method for the disk static data in iSCSI-SAN [19].

Sandor Acs et al, proposed an architecture for AoE(ATA-over-Ethernet) based storage support in OpenNebula cloud. This was implemented by novel storage solution and AoE based storage provides better (32.5$61.5 \%)$ compared to the prior iSCSI storage and the AoE storage solution requires less CPU time (41.3\%) to provide the service [20].

Tody Ariefianto W, Yudha Purwanto, they said, there is a need for iSCSI protocol optimization in order to improve the performance and reliability. They also analyze the iSCSI-SAN to handle the broken link. Implementation results shows that internet protocol multipathing enhance performance and reliability of iSCSI-SAN system [21].

Until emerging of a studies is new, included of an idea new for using iSCSI on the Storage visualization, that enable from using available storage disks storages. The communication between Initiator and Target devices in fully accordance with Initiator and Target model defines in iSCSI specifications not there any change, but change was on the storage type, to virtual storage.

Furthermore, the studies emerging were rounding about possibility that enable, from access to remote data that in available storage disks storages over IP- networked.

With storage technology of the SANs, and with using iSCSI-SAN protocol, for access to it. Where was the interest with the iSCSI-SAN network and FC-SAN network with respect to several component of a network protocol which impacts the performance of the network.

Where said Jiang Guo-song ,can worked on iSCSI out-of-band storage visualization design and implementation. The communication between Initiator and Target devices in fully accordance with Initiator and Target model defines in iSCSI specifications not there any change, but change was on the storage type, to virtual storage [22].

Adrian M. Caulfield and Steven Swanson et al, that are they described a paper's them with name QuickSAN although it's has other name, where describing QuickSAN a new SAN architecture designed for solid state memories. QuickSAN, a SAN prototype can be eliminates most software overheads and significantly reduces hardware overheads in SANs. QuickSAN integrates a network adapter into Solid State Disks (SSDs), so the SSDs can communicate directly with one another to service storage accesses as quickly as possible. With using QuickSAN can be reduces software and block transport overheads by between 82 and 95\% compared it FC and iSCSI-based SAN implementations and also demonstrated that QuickSAN can improve energy efficiency by $58 \%$ compared to a iSCSI-based SAN [4].

R. Purohit et al, proposed the SCVM, to creates iSCSI-SAN storage as virtual machine. The SCVM can fully manage and has features for the storage over local as well as networked VMs. This can be attained through virtual switch or physical network connection. It provides an advanced, fully featured iSCSI-SAN / Storage within an SAN Server [5].

A. Verma et al, clearly illustrated the performance analysis for SAN, for cost and complexity of FC. Where is based on several cases for SAN's performance analysis done with help of lometer tool and SAN's importance in clustered Database. In case of security FC-SANs are traditionally less secure 
than iSCSI. FC-SAN can see multiple Logical Unit Numbers(LUNs) on any particular disk, but iSCSI can only deal with a disk Target. Consequently, iSCSI authentication is very important, and iSCSI employs advanced authentication methods to establish security [3].

S. Hajirostam et al, the performance factors in SAN to figure out how to can improve the performance with respect to security solutions, where aimed to enhance the security level in SAN, with using compare between FC-SAN and iSCSI-SAN performance. The results, a model of iSCSI based SAN is simulated to measure the performance, and find out some of the security vulnerabilities and the solutions to make iSCSI-SAN secure and the storages the iSCSI-SAN has higher performance than FCSAN [2].

R. Jangid et al, explored the details of iSCSI and evaluated the performance of iSCSI-SAN with using iSCSI-SANs as virtual storage, for prove that iSCSI-SAN can be performed satisfactorily and economically, without requiring the costlier $\mathrm{FC}$ option. From the comparing between iSCSI-SAN and FC-SAN with respect to several component of a network protocol which impacts the performance of the network and Cluster mirroring with composition. iSCSI-SAN, improving reliability, high availability, management and high performance [6].

V. Aravindan, Where were the study about possibility for using the Storage server as a virtual SAN machines in the operating system of LINUX for better resource utilization [7].

\section{BACKGROUND}

\subsection{Concept of SAN}

Sometimes the terms DAS and SAN come up in topics regarding network storage. They are important in regards to NAS in that while these are separate concepts, DAS can be part of a NAS system and SANs can include NAS systems. In fact, applications generally cannot tell the difference between DAS storage and SAN storage [23]. This because of the application cannot really tell the difference between direct SCSI, iSCSI, or Fibre Channel, since all three are delivery SCSI block I/Os. Where in "fig. 3" ,the storage appears to host servers and applications in the same way that DAS storage appears, but because SAN storage uses a network, storage can be a long distance away from the host servers. SAN and DAS architectures use block SCSI protocol for sending and receiving storage data over their respective networks [25].

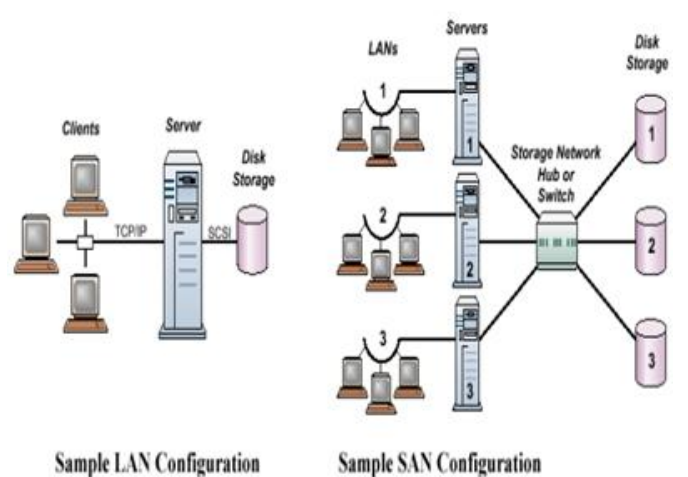

Fig. 3 The Sample Configuration of DAS and SAN [24].

\subsection{Definition}

The SAN is a network whose primary purpose is to transfer data between computer systems and storage elements [2]. A SAN is a high-speed architecture that interconnects remote storage devices with data servers. These storage devices are just like normal local attached devices, i.e., the users can do reads and writes operations with them. In SAN, the storage system is separated from the host. The storage devices form a local network and communicate with outside networks via a switch. Inside the SAN, users can easily add more storage devices without affecting the host. Any device failures may not harm the whole system. A SAN consists of servers, back-end storage devices and SAN connectors. There are two types of SAN, FC-SAN and iSCSI-SAN, according to the connecting media. Compared with DAS, a SAN can consist of more devices. Devices in a SAN are connected using FC or GbE which is faster than the SCSI cable in DAS [26]. As well as are networked infrastructures designed the SAN to beexcellent solution for a flexible, high-performance, highly scalable storage environment $[27,28]$. It is providing, the disaster-recovery, high data availability and business continuity, Synchronous storing or asynchronous storing enables customers to have an instantaneous recovery and eliminate loss of access to storage during disasters. Also, backups in the SAN network do not affect the rest of the LAN, since the back-up data passes only over the SAN, providing a LAN free back-up environment and less chances of network congestion 
occurred in frequent backups of data over the LAN, an instantaneous recovery and eliminate loss of access to storage during disasters [6].

\subsection{SAN Reasons}

The main reasons that make SAN a popular solution for storage networks are: disk utilization, disaster recovery methods, availability of data and fast backup data ability. SAN help users to use disk resources in a more efficient way, since all the disks in SAN are kept together as one resource so the management of disks become easier and disks can work better and more utilized, resulting in less waste of free space. This specific network can span a large number of systems(Microsoft Windows, UNIX, NT, Netware etc.) and geographic locations[2]. In addition, using any type of network (Ethernet, Fast Ethernet, Gigabit Ethernet), [31] as show in "fig. 4".

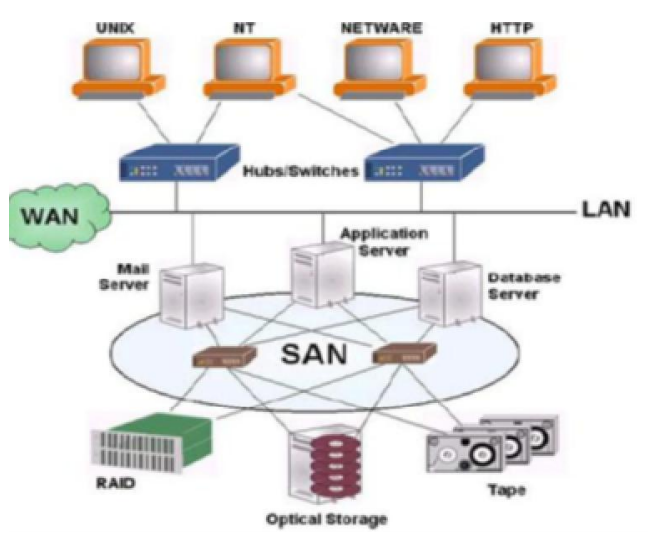

Fig. 4SAN [2].

\subsection{Architecture}

SAN architectures use block SCSI protocol for sending and receiving storage data over their respective networks. FC-SANs implement the SCSI protocol within the FC frames. iSCSI-SANs implement the same SCSI protocol within TCP/IP packets.SAN architecture provides a way to use block access methods over a network such as Ethernet or FC to provide storage for host computers. The storage in a SAN is not owned by one server but is accessible by all of the servers on the network. This SAN storage can be carved into logical storage pools or volumes that can be assigned to particular host servers. These logical volumes are independent of the geometries or components of the storage hardware [25].

\section{Generally, there are three types of networks :}

Home Area Networks (HANs), Business Area Networks (BANs) and Neighborhood Area Networks (NANs) [30].

SAN consists of a communication infrastructure, which provides physical connections, and a management layer, which organizes the connections, storage elements, and computer systems so that data transfer is secure and robust [31]. As shown in "fig. 4".

SAN, as mentioned in [3], allows direct, highspeed data transfers between servers and storage devices, potentially in any of the following three ways :

1 - Server to storage: This is the traditional model of interaction with storage devices. The advantage is that the same storage device might be accessed serially or concurrently by multiple servers.

2 - Server to server: A SAN might be used for high speed, high-volume communications between servers.

3 - Storage to storage: This outboard data movement capability enables data to be moved without server intervention, therefore freeing up server processor cycles for other activities like application processing.

Regardless of the method or topology used for transferring data, the terminology for the various parts of SANs is the same for all. Information is sent between "nodes", one of which is called the "transmitter" or "initiator" which is the source of the information and the receiving node is called a "receiver" or "target". Nodes can be any device that connects to a SAN that sends and receives information, such as a server or storage device [24].

\section{5. iSCSI-SAN Protocol}

As mentioned previously in paragraph 1 , After advent of iSCSI enables for small and medium businesses to reap the benefits of enterprise level FC storage solutions, without the expense of implementation and mandatory support expertise. Where the companies now have a cost effective storage solution that provides high performance, utilization of existing network infrastructures, and centralized storage [34]. This because of iSCSI is easy to understand. As shown in "fig. 5 " Instead of learning, building and managing two networks, an Ethernet LAN for user communication and a FC SAN for storage, an 
organization can use its existing Ethernet knowledge for both LAN and SAN, and also using of iSCSI in Ethernet IP environment is provides a lot of value in terms of skill sets, equipment, and commonality in services, capabilities and software tools. Where iSCSISAN can be implemented using NICs and switches that are running on the LAN now. Where iSCSI-SANs operate at 1 Gbps Ethernet speeds, though this could increase to $10 \mathrm{GbE}$ as NICs and switches are upgraded to accommodate $10 \mathrm{GbE}$ [10]. Additionally, iSCSI over TCP based SANs can extend over arbitrary distances, and are not subject to distance limitations that currently limit FCP [11], the Ethernet and TCP/IP compatibility of iSCSI-SANs extends their geographic location beyond the theoretical 10- kilometer maximum limit of FC to anywhere in the world where Internet connectivity is available. This offers tremendous flexibility in providing iSCSI-SAN availability to field offices, branch offices, and stranded servers.

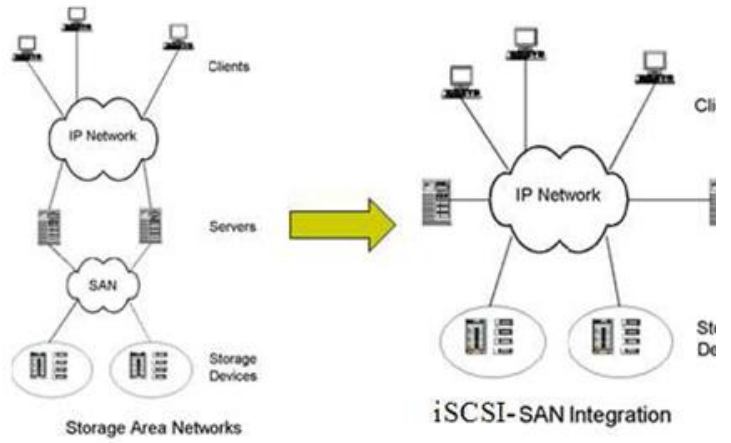

Fig. 5 : FC-SAN VS. iSCSI-SAN [11].

Moreover, it also allows virtually unlimited options in iSCSI-SAN location for optimum storage centralization and protection for disaster recovery. It also allows virtually unlimited options in iSCSI-SAN location for optimum storage centralization and protection for disaster recovery. At the same time iSCSI and FC-SANs can easily coexist. This allows legacy FC-SAN storage capacity to be readily expanded by using lower-cost, familiar Ethernet network components to add an iSCSI-SAN to an existing FC-SAN[33]. The iSCSI protocol uses the SCSI command set, on the networking side, the iSCSI uses TCP/IP and Ethernet protocol, where iSCSI encapsulates SCSI commands into TCP/IP packets and enables block-data transport over IP networks, for enabling application over a metropolitan and wide area networking as well [34].
Thus, iSCSI has the potential to enable blocklevel storage traffic to be accessed from a SAN over an IP network, and it can be expanded to virtually create a storage network by using any network-connected device [35].

The iSCSI architecture is similar to a client/server architecture or an Initiator/Target architecture. In this case, the client is an initiator that issues an $\mathrm{I} O \mathrm{O}$ request and the server is a target (such as a device in a storage system)[12]. i.e., iSCSI, has "two ends" in the connection, as shown in "fig. 6". These ends are the Initiator and the Target. iSCSI are callings them iSCSI Initiator and iSCSI Target. The iSCSI Initiator requests or initiates any iSCSI communication. It requests all SCSI operations like read or write. The Target is responsible for providing proper data requested by the Initiator and reporting to the Initiator completion of the data-transfer operation. The iSCSI architecture is using the TCP/IP connection to exchange SCSI commands. Where iSCSI is a protocol which encapsulates SCSI commands and data in TCP/IP packets for linking storage devices with servers over common IP infrastructures [13]. Where can be implemented between routers, host-to-switch, and storage array-tostorage array to provide asynchronous/synchronous data transfer [12].

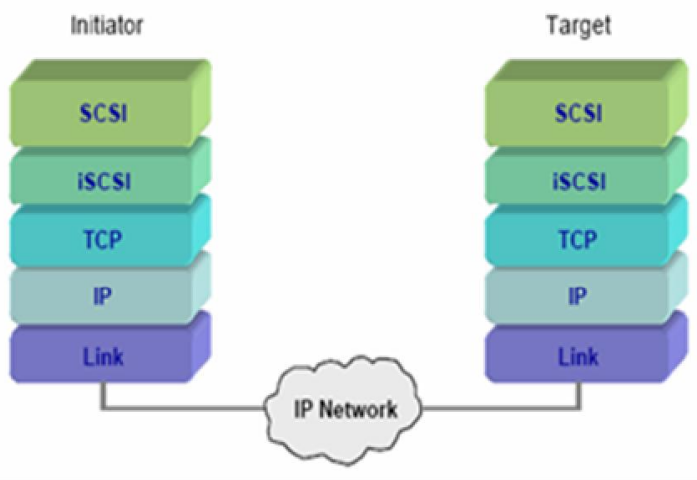

Fig. 6 : iSCSI-SAN Architecture [36].

The primary benefits of iSCSI, as was mentioned in [37]

>>No distance limitation

>>Lower cost

> Well understood technology

>>Easy to administer and manage

>>High availability (multiple connection paths) 
>>Better performance than NAS (block level access instead of file level)

>> Maximum utilization of resources (share disk and tape devices across a heterogeneous environment).

\section{PERFORMANCEEVALUATIONOF iSCSI-SANs}

In this section are explaining how can are to be rebuilt the prototype iSCSI-SANs as virtual storage with using attached storage disks storages DAS that in local system PC. Then using a iSCSI-SANs as storages in local system is rather than DAS storage in same local system. Can used the SCVM to is creating an iSCSI-SANs storage as virtual machine using iSCSI [7].

Experiment are explaining amount of the throughput, in terms of $(\mathrm{l} / \mathrm{Os}, \mathrm{MB} / \mathrm{s})$, and average response time in different block sizes of the $(\mathrm{I} / \mathrm{O})$ request, that can are achieves with using iSCSI-SANs as virtual with using storage technology of the SANs, and iSCSI protocol, and that can are achieves with using DAS which using SCSI protocol, in same system. Comparing of the amount of the throughput for iSCSI-SANs a, and DAS storage, To evaluate the performance which them are better, in local system PC.

Performance measurements, were made using the industry-standard $1 / O$ performance analysis and testing tool known as throughput in terms of $\mathrm{l} / \mathrm{Os}, \mathrm{MB} / \mathrm{s}$, and average response time in different block sizes of the $\mathrm{I} / \mathrm{O}$ request. Where:

- Throughput -IOPs: transaction rate in I/O operations per second (IOPS).

- Throughput-MB/s: throughput in megabytes per second $(\mathrm{MB} / \mathrm{s})$.

- Average response time in (ms).

The SCVM are application that creates iSCSISANs storage as virtual machine and is using as Storage Server, it provides an advanced fully, featured iSCSI-SANs. The first step that it must doing is creating a physical volume of iSCSI-SANs a single/more as virtual disk by SCVM, but before creating physical volume , the ISCSI target service is enables on KernSafe iStorage Server service tab, then creating physical volume from the disks that already attached and exist to PC system Where can creating volume in any size according to need. "Fig. 7" shows, can creating physical volume, with using the disk that already attached or exists in the local system, here creating one volume 10GB, where it use iSCSI file system.

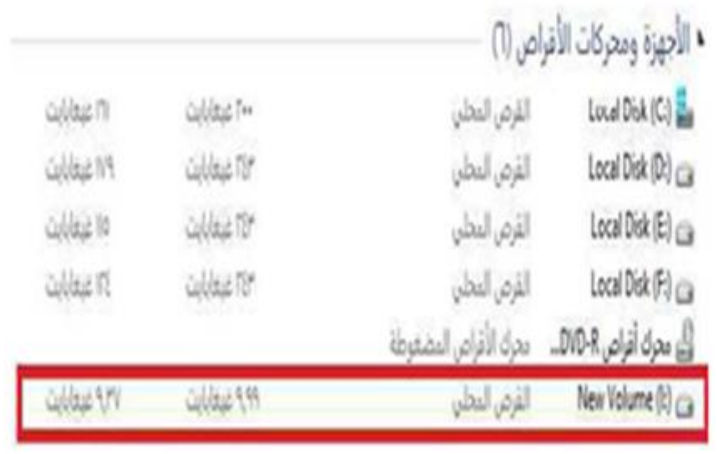

Fig. 7 Creating of iSCSI-SANs

After creating one volume 10GB that is using iSCSI file system in DAS as iSCSI-SANs. i.e., iSCSI-SANs are the attached storage disk DAS which done conversion it has, to be as virtual machine, to can for access to it by machines using iSCSI protocol. Experiment procedure for evaluates performance of iSCSI-SAN storage, with compare measures between iSCSI-SANs as SAN storage and DAS disk local in same system. For confirm that the iSCSI-SANs technology is well suitable to implement the SAN in local system rather than DAS storage in same local system.

The following steps describe the general experiment procedure implemented in this research work:

1. Start all services, like IOMeter and iSCSI software's on Initiator, Target and manager systems.

2. Connect each of the participating Initiators to its iSCSI Targets.

3. Login to the iSCSI Target using iSCSI by mounting all the storage devices available on the Target device from the Initiator.

4. Start the IOMeter, as shown in "fig. 8".

$>$ Specify name of disk test (I: New Volume) and (D: Local Disk)

$>$ Specify max disk size sectors and test connection value rate, as the following:

a. Maximum disk size 800000 sectors

b. Access specification: Default

c. Update frequency: 5 seconds 


\begin{tabular}{|c|c|c|c|c|r|r|}
\hline \multicolumn{7}{|c|}{ Table 1 Comparison between iSCSI-SANs and DAS } \\
\hline & \multicolumn{2}{|c|}{$\begin{array}{c}\text { Throughput (IOs) in T } \\
\text { time(ms) }\end{array}$} & \multicolumn{2}{|c|}{$\begin{array}{c}\text { Throughput-MBPS } \\
\text { in T Time(ms) }\end{array}$} & \multicolumn{2}{c|}{$\begin{array}{c}\text { Average Response in T } \\
\text { Time (ms) }\end{array}$} \\
\hline Blocksize & DAS & iSCSI-SANs & DAS & iSCSI-SANs & DAS & iSCSI-SANs \\
\hline $512 B$ & 1420.321 & 8343.825 & 0.693516 & 4.074133 & 42.63167 & 7.009223 \\
\hline 1 KB & 1413.626 & 8323.321 & 1.380494 & 8.128243 & 42.8558 & 7.033935 \\
\hline 2 KB & 1397.161 & 7905.385 & 2.728831 & 15.4402 & 43.22951 & 7.422626 \\
\hline 4 KB & 1483.642 & 7330.375 & 5.795478 & 28.63428 & 40.22635 & 7.997116 \\
\hline 8 KB & 1168.913 & 6513.17 & 9.132131 & 50.88414 & 51.32275 & 9.055521 \\
\hline $16 K B$ & 1089.597 & 5358.488 & 17.02496 & 83.72637 & 54.77454 & 11.00737 \\
\hline $32 K B$ & 666.7329 & 3815.82 & 20.8354 & 119.2444 & 89.46431 & 15.46689 \\
\hline $64 K B$ & 442.2056 & 2082.553 & 27.63785 & 130.1596 & 135.1781 & 28.15742 \\
\hline $128 K B$ & 221.542 & 1144.883 & 27.69275 & 143.1104 & 269.5271 & 51.73067 \\
\hline $256 K B$ & 110.8403 & 565.178 & 27.71007 & 141.2945 & 539.0964 & 105.3416 \\
\hline $512 K B$ & 55.36961 & 282.4949 & 27.68481 & 141.2475 & 1068.723 & 210.3243 \\
\hline $1024 K B$ & 27.67523 & 158.7016 & 27.67523 & 158.7016 & 2136.018 & 373.1266 \\
\hline
\end{tabular}

d. Run time: 6 minutes

5. Create the IOMeter.icf configuration files, and set the access specifications (request sizes) and the test setup parameter.

6. Start the test.

7. Name the output CSV file.

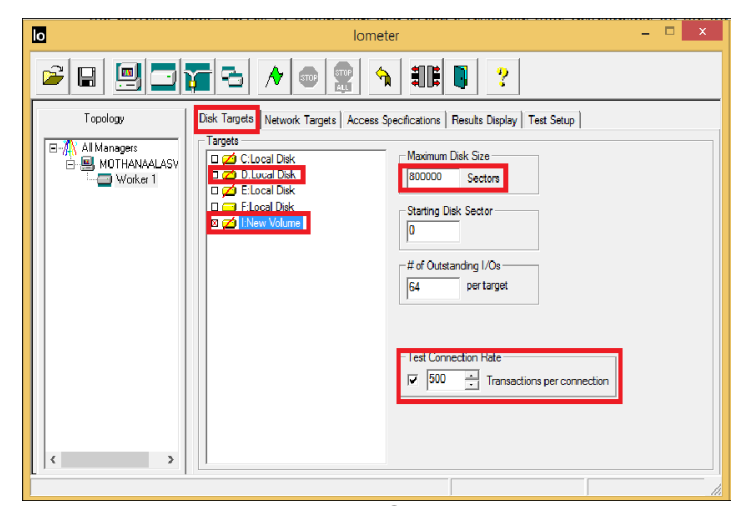

Fig. 8 IOMet

During procedure each the experiments, the IOMeter Initiators or clients made random read requests using different block sizes. For example, when performing the 512-byte request size, the worker process made random write requests for 512 bytes of data. The test was conducted using the following request sizes (in bytes) : 512B Reads, $1 \mathrm{~KB}$ Reads, 2KB Reads, 4KB Reads, 16KB Reads, 32KB Reads, 64KB Reads, 128KB Reads, 256KB Reads, 512KB Reads, 1024KB Reads. The results of the experiment are shown in table 1 , and the corresponding curves of Throughput and average response time dependence on the block size.

The results are represented graphically in "figs.9,10 and 11". The comparison of Throughput-IOPS in DAS and iSCSI-SANs with different packet size , is shown in "fig. 9". However, the comparison of ThroughputMBPS in DAS and iSCSI-SANs with different packet size, is shown in "fig. 10". Finally the comparison of average response time in different block sizes of the $1 / O$ request, in DAS and iSCSI-SANs, is shown in " fig. 11".

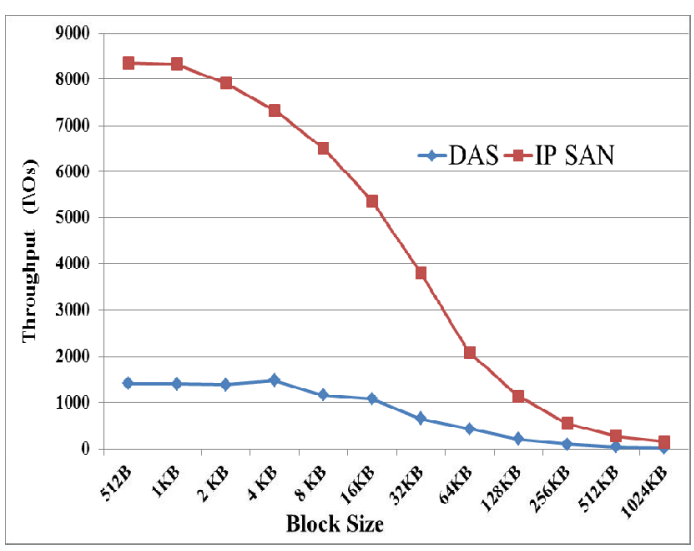

Fig. 9Throughput-IOPS in DAS and iSCSI-SANs. 


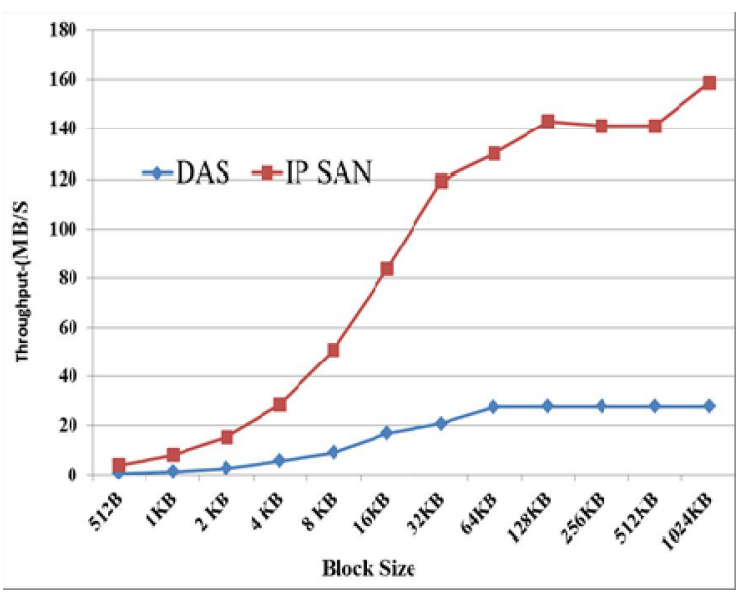

Fig. 10Throughput-MBPS in DAS and iSCSI-SANs.

Fig. 11Average Response Time in DAS and iSCSI-

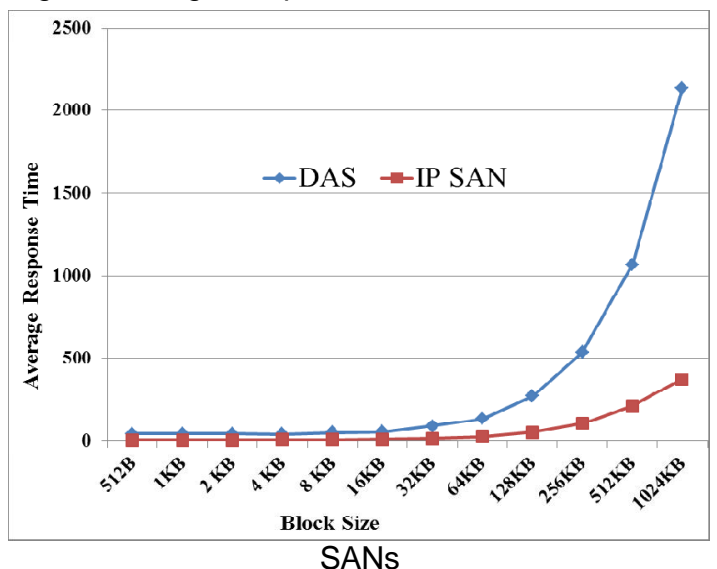

\section{CONCLUSION}

This paper studied of (SAN, iSCSI protocol) and investigates about possibility, using storage technology of the SANs, and iSCSISAN protocol, to improve access to attached storage disks storages in local system, with using iSCSI-SANs as virtual storage, is rather than DAS storage, in local system. Where explains the results of experiment is that improving throughput of iSCSI-SANs in terms of $(\mathrm{I} / \mathrm{Os}, \mathrm{MB} / \mathrm{s})$, and average response time in different block sizes of the $(\mathrm{I} / \mathrm{O})$ request, were better than attached storage disks storages as DAS in local system. This means that use iSCSI-SANs in local system as attaché storage disks storages as DAS , was become possible, without cost, high performance, easy control, and allow for use of all a traditional DAS at a fraction of the cost , without complexity and effort as SAN. The SCVM applications, allow for conversion DAS storage to an SAN storage as virtual machine are accessed by machines using iSCSI-SAN protocol. Thus, The iSCSI-SANs technology is well suitable to implement the SAN, until in system of PC same.

\section{Acknowledgements}

First of all I start by thanking Allah for assisting and guiding us in this work. I thank him for giving me the confidence and patience to finish thesis work.

I would like to express my appreciation and gratefully acknowledge my supervisor, Professor Doctor Khalil Alwagih, for his contributions to end this work, and to all Doctors in faculty of computer science \& information systems at Dhamar University.

\section{References}

[1] A. ZITOUNI, A. RECIOUI, A. SICHONE and A. SENOUSSAOUI "Design and Simulation of a WDM-OADM Optical Ring Communication System", Signals and Systems Laboratory, IGEE, UMBB University, 35000; Boumerdes, Algeria, Page range: 140-148, Vol.2, Issue 3, September-2017| ISSN-2543-3792.

[2] S. Haijirostam "Evaluation of Storage Area Network (SAN) Security and Performance", Master Thesis in Computer Network Engineering, Halmstad University, Sweden, November. 2013.

[3] A. Verma, S. Bhattacharjee, P. Baste, N. Mirajkar,S. Bhujbal, N. Deshmukh, K. Singh, "Performance analysis of Internet Protocol Storage Area Network (IP SAN) and its usage in Clustered Database", paper, For IJCSI International Journal of Computer Science Issues, Vol. 10, Issue 4, No 2, July 2013,ISSN (Print): 1694-0814,ISSN(Online):1694-0784, https://www.IJCSI.org . July. 2013.

[4] Adrian M. Caulfield , Steven Swanson, "QuickSAN: A Storage Area Network for Fast, Distributed Solid State Disks", Paper, University of California, USA, Apr. 2013.

[5] R. Purohit, R. Choudhary, "The Future of IP SAN using SCVM", International Journal of Smart Sensors and Ad Hoc Networks (IJSSAN) ISSN No. 2248-9738 Volume-1, Issue-3, 2012.

[6] R. Jangid, "Performance Evaluation of iSCSI based storage network with cluster mirroring", paper, For International Journal Of Engineering And Computer Science ISSN:2319-7242, Volume 3 Issue 3 March. 2014.

[7] V. Aravindan, "Performance analysis of an iSCSI block device in virtualized environment", Master of Science Thesis, TAMPERE University of Technology, October 2014.

[8] IOMeter Complete Guide. Iometer, performance analysis tool, Intel, http://csis.pace.edu/ lombardi/sciences/comp uter/systems/windows/docs/iometer.pdf, last modified 10 June. 2013 
[9] LI Bigang, SHU Jiwu, ZHENG Weimin, "SCSI Target Simulator Based on FC and IP Protocols in TH-MSNS", Paper, Department of Computer Science and Technology, Tsinghua University, Beijing 100084, China, October. 2006.

[10] S. J. Bigelow, iSCSI vs. fibre channel explained, fibre channel takes rightful place beside fibre channel,13 July 2007, http://www.cuttedge.com, July. 2007.

[11] Kalman Z. Meth and Julian Satran, "Design of the iSCSI Protocol" , IBM Haifa Research Laboratory, Haifa, Israel, Apr. 2003.

[12] Ron Dharma, Vinay Jonnakuti, Jonghoon (Jason) Jeong, "iSCSI SAN Topologies" , TechBook, Version 3.1, Copyright (C) 2011 2014 EMC Corporation, https://support.emc.com , 2014.

[13] Kalman Z. Meth and Julian Satran, "Design of the iSCSI Protocol" , IBM Haifa Research Laboratory, Haifa, Israel, Apr. 2003.

[14] C .M Gauger, M.Kohn, S.Gunreben, D.Sass and S. Gil Perez, "Modeling and Performance Evaluation of iSCSI Storage Area Networks over TCP/IP-based MAN and WAN networks" Broadband Networks, 2005. IEEE Transaction, vol 2, pp 850-858, Oct 2005.

[15] Chunhui Liu, "An Alternative Scalable Storage System" , Thesis , University Of Applied Sciences, 20.Nov. 2009.

[16] D. Zhang , "Performance Analysis of iSCSI Over TCP versus SCTP" , Thesis, Untversity Of Manitoba ,Canada, December. 2007.

[17] "Video Surveillance and External IP Storage Solutions" , paper. Technical Issues and Comparisons for IP SAN versus NAS Storage for Video Applications, (C) 2008 Intransa, Inc. All rights reserved, http://www.intransa.com, 2008.

[18] He Jun Wang Li, Li Yun-Shan, Tang lei Wen Chuan-hua, "Research and implementation of security iSCSI based on SSL" , Information and Computing (ICIC), 2011 Fourth International Conference, EEE Transaction, pp 18-21, Apr. 2011.

[19] Xu Xuedong, Su Jiyuan, "Research and Implementation of iSCSI based SAN Static Data Encryption System", Computer Science and Network Technology, 2nd International Conference, IEEE, pp 257 260, Dec. 2012.

[20] Sandor ACs, Mark Gergely, Peter Kacsuk, Miklos Kozlovszky, "Block Level Storage Support for Open Source laaS Clouds, Parallel, Distributed and Network-Based Processing (PDP)" , 2013 , 21st Euromicro International Conference, IEEE, pp. 262-268, Feb-March. 2013.

[21] Tody Ariefianto W, Yudha Purwanto, Hendra Wiratama, "Storage Area network Based-on Internet Small Computer Standard Interface Optimization Using Internet Protocol Multipathing" , Information and
Communication Technology (ICoICT), 2013 International Conference, IEEE, pp 303 307, March. 2013.

[22] Jiang Gao-song, He Xiao-ling, "Design and Implementation of iSCSI out-of-band storage virtualization", Intelligence Science and Information Engineering (ISIE), 2011 International Conference, IEEE, pp 378 381, Aug. 2011.

[23] Bill Griffee, "NETWORK ATTACHED STORAGE" , Thesis, Mikkeli University of Applied Sciences, May. 2013.

[24] Ashish A. Palekar, "Design And Implementation Of A Scsi Target For Storage Area Networks" , M.S. Thesis, University of New Hampshire, May. 2001.

[25] "Demartek iSCSI Deployment Guide 2011" , Demartek,http://www.demartek.com/iSCSI.htm I?iscsiguide, 2011.

[26] A. Pallampati, "iSCSI PERFORMANCE OVER RDMA-ENABLED NETWORK" , Thesis ,Wichita State University, Science Kansas (USA), 2006.

[27] R. Hernandez, C. Kion, K. Carmichae and G. Cole, "IP Storage Networking: IBM NAS and iSCSI Solutions" , Redbooks Publications (IBM), SG24-624001,http://www.storage.ibm.com/ibmsan, February. 2002.

[28] Vishvanath R, Azra Nasreen, "Survey on Recent Technology of Storage Area Network and Network Attached Storage Protocols" , Paper, For International Journal Of Innovative Research In Electrical, Electronics, Instrumentation And Control Engineering, Vol. 2, Issue 8, http://www.ijireeice.com, August. 2014.

[29] "easyRAID iSCSI Generic Software User Manual" , iSCSI GbE to SATA II / SAS, Disk Array System, Version 1.3, 2. Apr. 2008.

[30] Afaf Saoud and Abdelmadjid Recioui* , "A review on Data communication in smart grids" , Signals and Systems Laboratory, IGEE, UMBB University, 35000; Boumerdes, Algeria,Page range: 162- 179.

[31] J. Tate , P. Beck ,H. Hugo Ibarra, S. Kumaravel, M. than, "Introduction to Storage Area Networks and System Networking" , Libor 2012, IBM International Technical Support Organization. PDF document , http://www.redbooks.ibm.com/redbooks/pdfs/s g245470.pdf.Updated17,11.2012, $\quad$ Referred 25. March. 2013.

[32] Charles D. Jansen, "Storage Solutions Overview Benefits of iSCSI Implementation", Aberdeen White Paper, Published: December 2004, Copyright (C) 2004 ABERDEEN LLC, http://www.Aberdeeninc.com, December. 2004.

[33] "iSCSI Simplifies Storage Area Networks" , White Paper , Intel ® Server Adapters, 
Copyright @2007 Intel Corporation, http://www.intel.com, 2007.

[34] Federico Calzolari "“ High availability using virtualization " , Thesis, UNIVERSIT DIPISA ,in Italy ,Anno 2006.

[35] "Storage Networking with Fibre Channel \& IP Networks" , Copyright (C) 2004 Brocade Communications Systems, Inc. and Emulex Corporation, Availability http://www.emulex.com/, 2004.

[36] "Internet Protocol Storage Area Networks (IP SAN)" , Module 3.4, Copyright (C2006 EMC Corporation, All Rights Reserved, https://support.emc.com, 2006.

[37] "iSCSI Basics: A Practical Introduction " , Mosaic Technology Corporation, Salem, Availability, http://www.mosaictec.com. 